\title{
Study on Construction of a Power Station for the Intensive Breeding of Fur Animals
}

\section{Lang Xujun ${ }^{1, a}$, Wang Zhenhai²,b Chen Ming $^{2}$ Li Ziyang $^{1}$ Jin Ruii ${ }^{3}$ and Wang Ranran $3, \mathrm{c},{ }^{*}$}

${ }^{1}$ Shandong electric power engineering consulting institute corp. Itd, Jinan, Shandong, China

${ }^{2}$ State Grid Shandong Weifang Electric Power Company, Weifang, Shandong, China

${ }^{3}$ College of Mechanical and Electronic Engineering, Shandong Agricultural University, Tai'an, Shandong, China

a langxujun@sdepci.com, byiemin@126.com, wranran@163.com

\section{Keywords: Fur animals; Intensive farming; Power station; Electrical cooking; Electrical load}

Abstract. Fur intensive farming region due to the widely used feed electric processing equipment, makes the power peak increased dramatically during feeding time, the electric power planning and a new challenge to electric power dispatching. In this paper, the energy exchange of power plant and electric power system is proposed, and the cutting peak is reduced. This paper gives the calculation method of changing the service model of the power station and the capacity of the power station, and the actual effect of the change power station construction is verified through the concrete examples.

\section{Introduction}

With the high profits of fur-bearing animals farming from 1990s, the scales of fur-bearing animals farming are incessantly expanding and becoming relatively centralized. According to the statistics in Shandong, breeding stocks of fur-bearing animals summed up to 75 million in 2012, and in the areas of Zhucheng, Weifang, it amounted to 12.12 million, and ratio of mink's number was more than 95 percent. In China, fishes shrimps and poultry offal are mainly used for feeding fur-bearing animal as a traditional method [1-5]. The enzymes inside freshwater fish's body can destroy vitamin B, and proteins that poultry offal chiefly contains are uncompleted. So they all need to be thoroughly stewed before feed. Recently the stewing method of this sort of fodders is always heating in electric boilers, which are more environmental and convenient. But during the stewing time, it can cause successive load growth in power system, increases difficulty of grid dispatch operation and gives new challenges to the ability of power system regulation.

In the densely farmed regions of fur-bearing animals in China, demand of transportation increases with the extension of farming scale. Transport of feed and medicines needed in animals' breeding are also the main task of transportation besides transporting products used for sale. The characters of this kind of transportation are small traffic in one trip, not very large transportation range and high transporting frequency. If common agricultural mobiles or tractors are used in this transportation, consumption of energy would be extremely high and corresponding pollution would be serious, the efficiency would drop and cost of transportation would become comparatively high. However, electrical agricultural transport vehicles perfectly fit short range, small traffic and dispersive transportation, and they have advantages of high mobility, low emission, low cost and convenient [7, 8]. But low endurance mile and lack of related supporting facilities make electrical agricultural transport vehicles hard to be generalized.

This paper gives a new way that uses power station to generalize the application of electrical agricultural transport vehicles, and solve problems in power system dispatching operation at the same time. Due to the concentrated distribution of batteries waiting for charging in power station and the convenient general dispatch and flexible distribution of charging power, the difference between peak and valley in grid load and fluctuation of load can be eliminated by controlling charging or discharging of power station. Meanwhile this paper builds charging optimized model for minimum 
charging cost. Replaceable power battery reserve can be formed by organic allocation of battery charging and distribution network supply. On the base of guarantee in power exchanging service availability and adequacy of power exchanging in power station, problems of power tension and drop of power quality from power peak are effectively adjusted when concentrated utility of power among individuals results in dramatic increase of short-term load.

\section{System structure and function model}

The power station system in this paper consists of grid connection units, conversion units, central control system, power battery charging, and exchanging units and so on, which are shown in figure 1.

Power battery charging and exchanging units includes power battery system and corresponding DC-DC converter modules. Power station exchanges power battery for electrical agricultural vehicles which have need of exchange. In the same time, power supply system in power station gets these disassembled batteries charged.

Charging process of power battery usually has 3 stages, which is constant current charging at first, then constant voltage charging, finally becomes floating charging. Thus in the charging period of single power battery, power output will not be a constant. But in the process of charging, constant current charging stage generally has fastest speed of SOC growth. Hence as far as charging and discharging power distribution concerned, average charging power is adopted in describing single power battery charging requirements to avoid trivial calculation.

Battery packs used in electrical agricultural transport vehicles are generally $72 \mathrm{~V} 110 \mathrm{Ah}$ at present, and sum up to about $7.9 \mathrm{~kW}$. They are mainly lead-acid batteries. To assure long life and charging fullness of battery, charging current selects $0.15 \mathrm{C}$. Exchanging time regularly and equably distribute in 23:00 5:30 and 10:00 16:30.

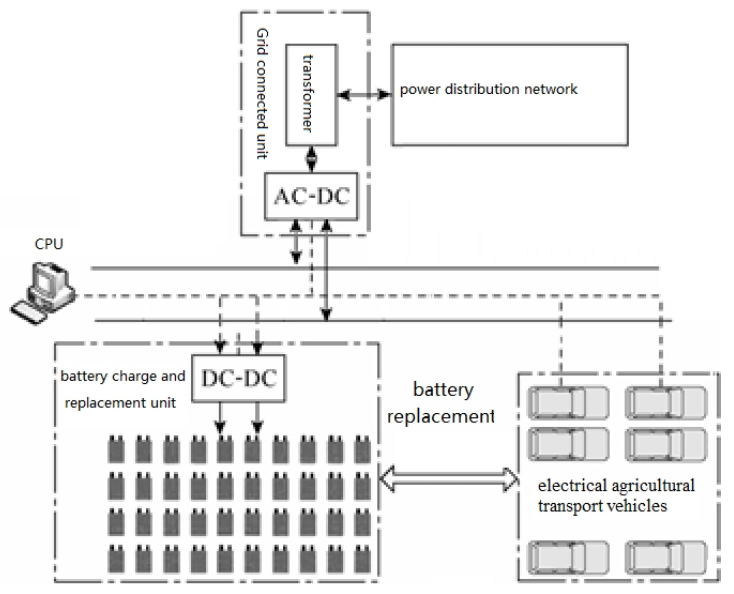

Fig. 1 Power station system structure

\section{Exchanging service model and calculation of battery reserve}

Exchanging power station is a kind of special load with controllability; operators can reasonably choose charging time periods, number of charging batteries in every time period according to the price in peak or valley stage, and guarantee the number of charging battery packs as many as possible with valley price while satisfying exchanging power requirements in every time period. Battery packs can be arranged to charge in proper time and devoted into use. Battery packs will be prompted to be fully circularly used on the base of the satisfaction of exchanging power requirements; the redundancy of battery packs will not be too high or too low.

To simplify the calculation, suppose that battery packs are charged with constant power $\mathrm{P} \mathrm{kW} / \mathrm{h}$ from empty to fullness every time. And disassembled that empty battery packs are arranged to be charged from the initial moment of a random time stage.

Capacity optimization configuration model is an integer nonlinear programming function.

$$
\min F=\gamma_{\mathrm{s}} \mathrm{f}_{\mathrm{es}}(\mathrm{P})+\mathrm{f}_{\mathrm{wh}}(\mathrm{P})+\gamma_{\mathrm{h}} \mathrm{f}_{\mathrm{eb}}+\mathrm{f}_{\mathrm{ed}}
$$

$\mathrm{F}$ is total expense, $f_{\mathrm{es}}(P)$ represents expense of purchasing charging facilities in planning period, $\gamma_{\mathrm{s}}$ is annual average expense coefficient of charging facilities. $f_{\text {wh }}(P)$ represents annual charging facilities maintenance expense. $f_{\text {eb }}$ is expense 
of purchasing battery packs in planning period. $\gamma_{\mathrm{b}}$ represents annual average expense coefficient of battery packs. $f_{\text {ed }}$ is annual purchasing power expense in operating stage. $f_{\mathrm{es}}(P)$ and $f_{\mathrm{wh}}(P)$ are functions of exchanging power station capacity $P$.

Exchanging power station capacity $\mathrm{P}$ is the linear function of charging facilities number. It means $\mathrm{P}$ generally confirmed by charging facilities number. Capacity programming equals programming of charging facilities in station. So equation (1) can be expressed as:

$$
\min F=N_{S}\left(\gamma_{S} f_{S 0}+f_{S 0}\right)+\gamma_{B} N_{B} \cdot p_{B}+365 \sum_{T=1}^{24} M_{T} P_{C} p_{T}
$$

In this equation, $N_{\mathrm{s}}$ is number of charging facilities, $f_{\mathrm{s} 0}$ and $f_{\mathrm{h} 0}$ represents single charging facility purchasing expense and maintenance expense respectively. $N_{\mathrm{B}}$ is number of purchasing charging battery packs in initial period. $p_{\mathrm{B}}$ is single battery pack price. $M_{\mathrm{T}}$ is the number of battery packs in charging in period T. $P_{\mathrm{C}}$ is single battery pack charging power. $p_{\mathrm{T}}$ is power price in period T. $p_{\mathrm{f}}, p_{\mathrm{p}}, p_{\mathrm{g}}$ is peak, flat and valley price separately. $n_{\mathrm{f}}, n_{\mathrm{p}}, n_{\mathrm{g}}$ is the number of peak, flat and valley period separately. $M_{\mathrm{fT}}, M_{\mathrm{pT}}, M_{\mathrm{gT}}$ is charging battery packs number in the period of peak, flat and valley price separately.

Charging constraint condition: suppose that battery packs charging time has $T_{\mathrm{b}}$ time periods. Battery packs can be subdivided into $T_{\mathrm{b}}$ charging processes in the first $\mathrm{T}$ period. Each charging process number of battery packs under the charging situation can be expressed as $\mathrm{M}_{\mathrm{T}}^{\mathrm{Tb}}$, and satisfies the following constraint equation:

Charging battery packs number balance constraint equation:

$$
\mathrm{M}_{\mathrm{T}}^{\mathrm{n}}=\mathrm{T}_{\mathrm{T}+1}^{\mathrm{n}+1}
$$

$$
\mathrm{N}+\mathrm{X}_{\mathrm{T}}+\mathrm{J}_{\mathrm{T}}+\mathrm{K}_{\mathrm{T}}+\sum_{\mathrm{n}=1}^{3} \mathrm{M}_{\mathrm{T}}^{\mathrm{n}}=\mathrm{N}_{\mathrm{B}}
$$

$N$ is self-carrying charging battery packs number of electrical agricultural transport vehicles. $J_{\mathrm{T}}$ is battery packs number with full power in period T. $K_{\mathrm{T}}$ is empty battery packs number in period T.

Constraint equation of exchanging demand in exchanging power station is:

$$
\sum_{\mathrm{n}=1}^{3} \mathrm{M}_{\mathrm{T}}^{\mathrm{n}} \mathrm{P}_{\mathrm{C}}=\lambda_{\mathrm{T}} \mathrm{N}_{\mathrm{S}} \mathrm{P}_{\mathrm{tr}}
$$

$\lambda_{\mathrm{T}}$ is state efficiency when charging facilities are working. $P_{\mathrm{tr}}$ is required power when single charging facility fully works.

\section{Power station case and analysis}

A village has 300 residents, each resident is calculated in $4.5 \mathrm{~kW}$, total power consumption multiplied by power utility coefficient $40 \%$, then the daily load curve of power consumption in illumination is shown in figure 2 , peak value is $540 \mathrm{~kW}$, peak to valley value is $507.6 \mathrm{~kW}$.

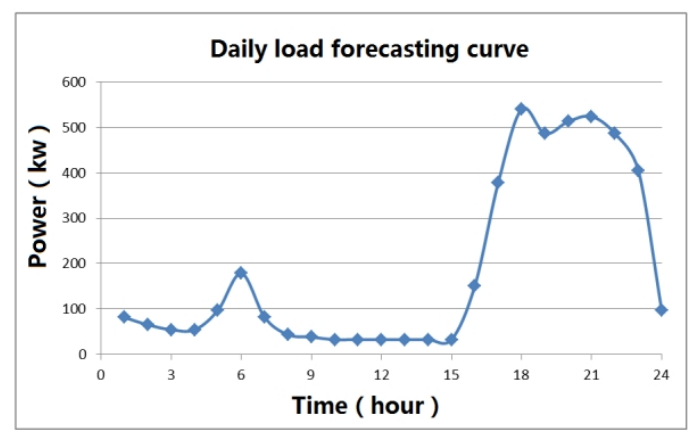

Fig.2 Daily load curve of power consumption in illumination

Intensive farming village has $30 \%$ residents, which are 60 residents farming minks. Assume that main transport tool is electrical agricultural transport vehicles. There are at least 100 such vehicles in this village and power consumption of each electrical agricultural transport vehicle is $8 \mathrm{kWh}$ at present. To make sure the quality of batteries, charging current selects $0.15 \mathrm{C}$, and batteries reach fullness in $10 \mathrm{~h}$, According to the habits of using agricultural vehicles, electrical agricultural vehicles should be charged during 19:00 pm and 5:00 am next day. Consider the daily load curve of electrical agricultural vehicles and this curve is shown in figure 3, peak value is $643.8 \mathrm{~kW}$, peak to valley value is $611.4 \mathrm{~kW}$. 


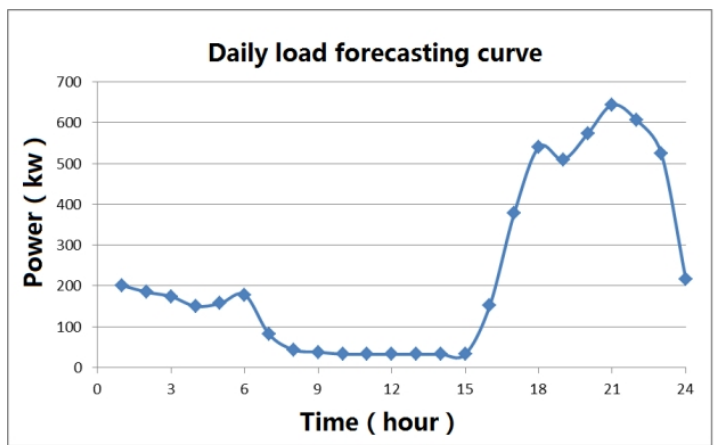

Fig. 3 Daily load curve of electrical agricultural vehicles

Adult minks are fed twice a day (9:00 and 17:00), the power of a meat grinder is generally 11 15kW, power of an electric boiler is about $10 \mathrm{~kW}$ and it is operated in about $2 \mathrm{~h}$. Then the daily load curve can be shown in figure 4 . Peak value is $1140 \mathrm{~kW}$; peak to valley value is $1107.6 \mathrm{~kW}$.

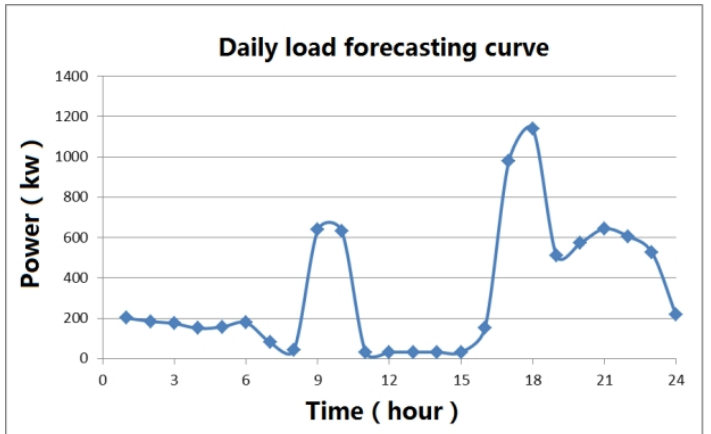

Fig. 4 Daily load curve of power consumption in minks breeding

On the condition of unchanged minks feed habit, electrical agricultural vehicles free charging is replaced into regular charging by building power station. According to the calculation of power station, retain 500kW discharging ability in high power peak period, discharge with the ability of $200 \mathrm{~kW}$ in 9:00 and 10:00, 400kW in 17:00 and 500kW in 18:00. The daily load curve is shown in figure 5 , peak value is $523.8 \mathrm{~kW}$, and peak to valley value is $486 \mathrm{~kW}$.

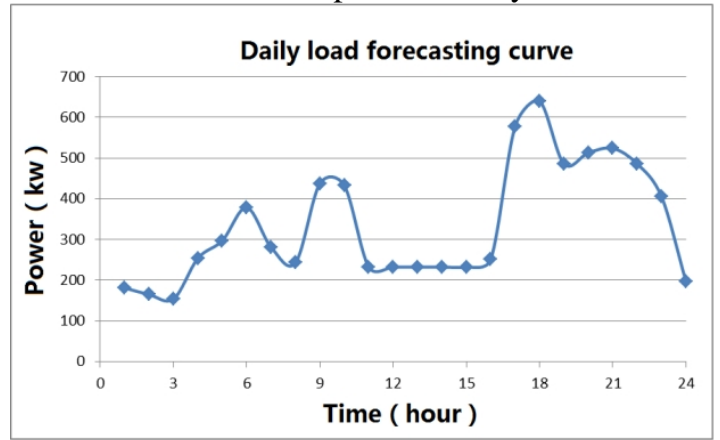

Fig. 5 daily load curve with adjustment of power station

\section{Conclusions}

The establishment of power station makes power batteries not only gotten charged as loads from grid but also become an effective method of power system load adjustment and gotten involved into the power dispatch. When the power station is under planning, grid electricity purchasing cost in the operation period should be taken into account so that response from charging time in the centralized charging selection to the mechanism can be realized. While the profits of operators are increased, reduction of difference between peak and valley can be achieved. So the safety of grid operation is effectively enhanced and the economy of power operation with less waste of social resources can be guaranteed. While power planning is optimized, the development of electrical agricultural transport vehicles is propelled, not only the energy conservation and emission reduction is realized but also the demand of multifunction and super miniature in country labors can be met. Research in this paper fit fur-bearing animal intensive farming regions and also lay theoretical foundation of power station establishment in the regions with high difference of power load.

\section{Acknowledgements}

This work is supported by Project of Shandong electric power engineering consulting institute corp. (37-K2016-059). 


\section{References}

[1]Wenli Li, Taiyue Yin, Xingchen Li, Mei Lü, Huiying Ren. General Situation of Fur Animal Mixed Breed Development in Shandong Province[J]. Journal of Economic Animal, 2015, 19(03):167-171.

[2]Jingchao Yang, Xuxian Qu, Zefang Ma, Youzhi Li, Kaifeng Zhou. Development Status and Countermeasures of Fur Animal Breeding Industry in Shandong Province[J]. Journal of Economic Animal, 2013, 17(01):41-44.

[3]Guizhi Wang, Zijuan, Qin, Wei Zhang. Problems and Countermeasures of the Present Situation of Fur Animal Breeding Industry in Shandong Province[J]. Shandong Journal of Animal Science and Veterinary Medicine, 2014, 35(08):21-22.

[4]Shanshan Zhao, Qingjie Pan, Lihua Wang. Investigation on the Situation of Mink Breeding in Zhucheng District, Shandong Province[J]. Heilongjiang Animal Science and Veterinary Medicine, 2015, (22):150-152.

[5]Chao Yu, Wei Wang, Miao Qian. The Present Situation, Restricting Factors and Development Analysis of Mink Breeding Industry in Zhucheng District[J]. Heilongjiang Animal Science and Veterinary Medicine, 2015, (20):14-17.

[6]Chengzhi Zhang. The Notices of Mink Feeding Animal Feed[J]. Feed Review, 1990, (04):46.

[7]Xiao Sun, The Development Status and Trend of Light Electric Agriculture Transportation Machinery[J]. Agricultural Development \& Equipments, 2012, (03):49-50.

[8]Xuejiao Shen, Jiajie Feng. Agricultural Vehicle Market Prospect Analysis[J]. Fujian Quality Management, 2016, (02):86.

[9]Shu Zhang, Zechun Hu, Yonghua Song, Masoud Bazargan. Study on the Combination of Electric Vehicle Changing Power Station and Power Grid Interaction [J]. Proceedings of the CSEE, 2012, 32(10):8+49-55. 